\title{
Aplicando a Experimentação na Engenharia de Requisitos: O Caso do Método ERi*c
}

\author{
Antonio de Padua A. Oliveira ${ }^{1}$, Julio Cesar Leite ${ }^{2}$, Luiz Marcio Cysneiros ${ }^{3}$ \\ ${ }^{1}$ Departamento de Informática - Universidade do Estado do Rio de Janeiro (UERJ) \\ Rua São Francisco Xavier, 524 - Sala 6019 - CEP 20559-900 - RJ - Brazil \\ 2 Departamento de Informática - Pontificia Universidade Catolica do Rio de Janeiro \\ (PUC-Rio) - Rua Marques de São Vicente, 225 - CEP 22453-900 - RJ - Brazil \\ ${ }^{3}$ School of Information Technology - York University, (YorkU) \\ Toronto - Ontario - Canada \\ padua@ime.uerj.br, julio@inf.puc-rio.br, cysneiro@yorku.ca
}

\begin{abstract}
Some researches in Software Engineering said that only experiments in the centre of scientific process can verify new theories and point to needed corrections and to explore critical factor of these new theories. They also said that new methods, techniques and tools in Software Engineering should not be published without before be validated and compared with others that are working. This work shows three experiments application, which had the aim of evaluating ERi*c Method competencies. The Method ERi ${ }^{*} c$ was developed in the doctoral thesis named Intentional Requirements Engineering: A Method for Requirements Elicitation, Modeling, and Analysis.
\end{abstract}

Resumo. Alguns pesquisadores em Engenharia de Software afirmam que somente os experimentos, no centro do processo cientifico, podem verificar novas teorias e, indicar as correções cabiveis e explorar os fatores críticos dessa nova teoria. Afirmam também que novos métodos, técnicas e ferramentas em Engenharia de Software não deveriam ser publicados sem antes serem experimentados, validados e comparados com os existentes. Este trabalho apresenta a aplicação de três experimentos, os quais tiveram com o objetivo avaliar as competências fornecidas pelo Método ERi ${ }^{*} \mathrm{c}$ da tese de doutorado intitulada: Engenharia de Requisitos Intencional: Um Método de Elicitação, Modelagem e Análise de Requisitos.

\section{Introdução}

Segundo o trabalho "Introdução a Engenharia Experimental" [Travassos, 2002], para a Engenharia de Software existem quatro métodos relevantes com a finalidade de condução de experimentos. São eles: (1) o método científico que retira do ambiente observado um modelo para definir o conjunto de propriedades que devem ser analisadas; (2) o método da engenharia que estuda as soluções já utilizadas e aplica alguma suposta evolução na tentativa de achar uma vantagem adicional em relação às soluções iniciais; (3) o método analítico, ou matemático, que propõe uma teoria bem formalizada, obtém os resultados da aplicação da mesma e compara esses resultados com os resultados empíricos e (4) o método experimental que propõe e submete 
repetidamente o novo método a situações para observação do comportamento com o objetivo de comprovação e aprimoramento.

Travassos [Travassos, 2002] afirma que somente experimentos, como o centro do processo científico, podem verificar novas teorias e, dessa maneira, indicar as correções cabíveis e explorar os fatores críticos dessa nova teoria. Travassos vai mais além quando afirma que novos métodos, técnicas e ferramentas em Engenharia de Software não deveriam ser publicados sem antes serem experimentados, validados e comparados com os existentes. Mas, complementando, Travassos ressalva que nenhum experimento pode levar a uma evidência absoluta e cita Basili [Basili, 1996], que apresenta com detalhes quais são os propósitos e as vantagens da experimentação na Engenharia de Software.

Nas próximas seções apresentamos a aplicação dos procedimentos da experimentação, segundo o processo recomendado por Travassos et al. [Travassos, 2002] em "Introdução à Engenharia Experimental". Detalhamos os objetivos a serem atingidos, as questões a serem respondidas e as métricas utilizadas, de acordo com o método GQM - "Goal, Question, Metric" [Basili, 1994]. Também detalhamos o planejamento do processo e ao final apresentamos a operacionalização do processo através dos questionários que o apoiaram. A última seção apresenta um resumo dos resultados obtidos.

\section{Definição Sucinta do Processo de Experimentação}

Para avaliar e validar o método Engenharia de Requisitos Intencional - ERi*c [Oliveira 08a] foi aplicado o "Processo de Experimentação em Engenharia de Software" obtido em [Mafra, 2006], que foi definido por Wohlin et al. (2000) e estendido por Amaral (2003). O Processo de Experimentação possui dois subprocessos, o Processo de Execução e o Processo de Empacotamento.

As finalidades das etapas do Processo de Experimentação são apresentadas a seguir. A etapa de definição vai expressar o experimento em termos de objetivos e problemas; a etapa de planejamento vai: determinar o projeto do experimento, definir a instrumentação a ser utilizada e analisar os aspectos de validação dos resultados. A etapa de execução vai cuidar da coleta dos dados do experimento. E a etapa de análise e interpretação vai estudar detalhadamente os dados coletados. O processo possui dois pontos de controle, o primeiro existe para avaliar a necessidade de replanejar e o segundo existe para avaliar a efetividade da etapa de análise dos resultados. O Processo de Empacotamento faz a atividade de organizar e guardar as informações a respeito do Processo de Execução.

\section{Os Estudos da Experimentação}

A motivação envolvida pelo processo de experimentação que executamos foi guiada pela idéia de avaliar se o Método ERi*c ajuda as competências do Engenheiro de Requisitos (ER).

Para a avaliação das competências fornecidas precisaríamos comparar o Método ERi*c com o Framework i* [Yu, 1995] já que o Método ERi*c se propõe a suprir a lacuna da elicitação de metas que ainda não está equacionado pelos métodos GORE, inclusive pelo Framework i*, como também contribuir para a redução dos problemas de 
escalabilidade do Framework i* [Yu, 1995] relatados por Pastor et al. [Pastor, 2006] e por Estrada et al. [Estrada, 2006]. Conseqüentemente, seguindo o processo de experimentação de Mafra e Travassos [Mafra, 2006] nossa etapa de planejamento previa a realização de duas experimentações. A primeira devia avaliar se as competências atribuídas ao Framework i* eram úteis e se estavam adequadamente definidas segundo o ponto de vista de engenheiros de requisitos em relação às competências de métodos já dominados, ou já ensinados como Análise Essencial e Orientação a Objetos. A segunda devia comparar as competências fornecidas pelo Método ERi*c com as competências atribuídas pelo Framework i* [Yu, 1995]. Porém, nosso planejamento inicial foi alterado, incluímos mais uma experimentação. Influenciados pela necessidade do "pôster" [Oliveira, 2008b] aceito no CAISE 08, avaliamos separadamente se a estratégia de diagnósticos "i* Diagnoses" trazia competências úteis, e com a definição adequada, em relação às etapas anteriores do próprio Método ERi*c.

O objetivo da experimentação foi único. Mas, a experimentação foi desmembrada em três para que a avaliação dividida pudesse trazer, apesar do trabalho triplo, mais retorno de informação para a tese de [Oliveira, 2008a]. A próxima seção apresenta as etapas do estudo das competências e sua aplicação nos três experimentos realizados.

\section{O Estudo das Competências do Método ERi*c e do Framework i*}

\section{ETAPA DE DEFINIÇÃO}

\section{I.1 Objetivo global}

O objetivo global da experimentação do método Engenharia de Requisitos Intencional ERi*c é: "Estabelecer quais são as vantagens fornecidas pelo Método ERi*c em relação ao Framework $i *$, em termos de competências para a Engenharia de Requisitos de Sistemas Multi-Agentes, na elicitação, na modelagem e na análise dos requisitos".

\section{I.2 Objetivos da medição}

Os objetivos da medição, guiados pelo processo, devem identificar:

(i) Quais são as competências adquiridas?

(ii) Quais são as competências consideradas úteis?

(iii) Quais são as competências consideradas inúteis?

(iv) Quais são as competências em potencial?

\section{I.3 Objetivos do estudo}

Identificar se o método proposto Engenharia de Requisitos Intencional - ERi*c, contribui para melhorar as "competências" atribuídas à Engenharia de Requisitos de Sistemas Multi-Agentes do ponto de vista dos engenheiros de requisitos. 


\section{I.4 Questões e métricas}

Com a idéia de aplicar a abordagem GQM [Basili, 1994] para a avaliação do objetivo global definido no parágrafo 1.1 anterior definimos nos parágrafos a seguir os objetivos (Goals), as perguntas investigadas (Questions) e as métricas (Metrics) da abordagem.

GOAL: "Estabelecer quais são as vantagens fornecidas pelo Método ERi*c em relação ao Framework i*, em termos de competências para a Engenharia de Requisitos de Sistemas Multi-Agentes".

Q1: Quais competências são fornecidas pelo Método ERi*c e não fazem parte do Framework i*?

$\Rightarrow$ Métrica: Lista das competências fornecidas pelo Método $\mathrm{ERi}^{*} \mathrm{c}$ que não fazem parte do Framework $i^{*}$.

Q2: Quais competências são fornecidas pelo Método ERi*c e são consideradas inúteis pelos engenheiros de requisitos?

$\Rightarrow$ Métrica: Lista das competências fornecidas pelo Método ERi*c e que são consideradas inúteis pelos engenheiros de requisitos.

Q3: Quais competências são fornecidas pelo Método $\mathrm{ERi}^{*} \mathrm{c}$ e fazem parte do Framework $i^{*}$ e são consideradas úteis pelos engenheiros de requisitos, cujo detalhamento deve ser modificado?

$\Rightarrow$ Métrica: Lista das competências fornecidas pelo Método $\mathrm{ERi}^{*} \mathrm{c}$ que fazem parte do Framework $i^{*}$ e são consideradas úteis pelos engenheiros de requisitos, cujo detalhamento deve ser modificado.

Q4: Quais competências são fornecidas pelo Método ERi*c e não fazem parte do Framework i*, mas que são consideradas úteis pelos engenheiros de requisitos?

$\Rightarrow$ Métrica: Lista das competências fornecidas pelo Método ERi*c que não fazem parte do Framework $i^{*}$, mas que são consideradas úteis pelos engenheiros de requisitos.

\section{ETAPA DE PLANEJAMENTO}

\section{II.1 Definição das hipóteses}

A HIPÓTESE, para acompanhar o desmembramento da experimentação, teve que ser também desmembrada e, assim, enunciada em separado para cada estudo executado. A INSTRUMENTAÇÃO e a SELEÇÃO DO CONTEXTO dos experimentos foram definidas apenas uma vez porque elas não sofreram mudança. Por outro lado, a DEFINIÇÃO DAS VARIÁVEIS dos experimentos precisou ser redefinida para cada um dos experimentos. 


\section{II.1.1 Definição das hipóteses para a experimentação 1}

Hipótese nula $\left(\mathbf{H}_{0}\right)$ : As competências fornecidas pelo método Framework $i^{*}$ são equivalentes às competências anteriormente assimiladas.

C1 - conjunto de competências fornecidas pelo Framework i*

C0 - conjunto de competências anteriormente assimiladas

$$
\mathrm{H}_{0}: \mathrm{CO}-(\mathrm{C} 1-\mathrm{CO})=\varnothing
$$

Significa que não existem competências fornecidas pelo Framework $i^{*}$ que não fazem parte das competências anteriormente assimiladas.

Hipótese alternativa $\left(\mathbf{H}_{1}\right)$ : As competências fornecidas pelo Framework i* não são equivalentes às competências anteriormente assimiladas.

C1 - conjunto de competências fornecidas pelo Framework i*

C0 - conjunto de competências anteriormente assimiladas

H1: $\mathrm{CO}-(\mathrm{C} 1-\mathrm{CO}) \neq \varnothing$

Significa que existem competências fornecidas pelo Framework $i^{*}$ que não fazem parte das competências anteriormente assimiladas.

Hipótese alternativa $\left(\mathbf{H}_{2}\right)$ : $\mathrm{Na}$ lista das competências fornecidas pelo Framework $\mathrm{i}^{*} \mathrm{e}$ que não fazem parte das competências anteriormente assimiladas existem competências que são consideradas úteis pelos engenheiros de requisitos, cujo detalhamento deve ser modificado.

$\mathbf{C x}$ - conjunto de competências fornecidas pelo Framework $i^{*}$ e que não fazem parte das competências anteriormente assimiladas.

Cy - conjunto de competências que são consideradas úteis pelos engenheiros de requisitos, cujo detalhamento deve ser modificado.

H2: $\mathbf{C y}-(\mathbf{C x}-\mathbf{C y}) \neq \varnothing$

Significa que existem competências fornecidas pelo Framework $i^{*}$ que não fazem parte das anteriormente assimiladas, mas são consideradas úteis pelos engenheiros de requisitos e cujo detalhamento deve ser modificado.

\section{II.1.2 Definição das hipóteses para a experimentação 2}

Hipótese nula $\left(\mathbf{H}_{\mathbf{0}}\right)$ : As competências fornecidas pelo Método $\mathrm{ERi}^{*} \mathrm{c}$ são equivalentes as competências fornecidas pelo Framework $i^{*}$.

C1 - conjunto de competências fornecidas pelo Método ERi*c

C0 - conjunto de competências fornecidas pelo Framework $i^{*}$

H0: $\mathrm{CO}-(\mathrm{C} 1-\mathrm{CO})=\varnothing$ 
Significa que não existem competências fornecidas pelo Método ERi*c que não fazem parte do Framework i*.

Hipótese alternativa $\left(\mathbf{H}_{1}\right)$ : As competências fornecidas pelo Método ERi*c não são equivalentes as competências fornecidas pelo Framework i*.

C1 - conjunto de competências fornecidas pelo Método ERi*c

CO - conjunto de competências fornecidas pelo Framework i*

$\mathrm{H}_{1}: \mathrm{CO}-(\mathrm{C} 1-\mathrm{CO}) \neq \varnothing$

Significa que existem competências fornecidas pelo Método $\mathrm{ERi}^{*} \mathrm{c}$ que não fazem parte das competências fornecidas pelo Framework $i *$.

Hipótese alternativa $\left(\mathbf{H}_{\mathbf{2}}\right): \mathrm{Na}$ lista das competências fornecidas pelo Método ERi*c e que não fazem parte das competências fornecidas pelo Framework $i^{*}$ existem competências que são consideradas úteis pelos engenheiros de requisitos, cujo detalhamento deve ser modificado.

$\mathbf{C x}$ - conjunto de competências fornecidas pelo Método ERi*c e que não fazem parte das competências fornecidas pelo Framework i*.

Cy - conjunto de competências que são consideradas úteis pelos engenheiros de requisitos, cujo detalhamento deve ser modificado.

$$
\mathrm{H}_{2}: \mathbf{C y}-(\mathbf{C x}-\mathbf{C y}) \neq \varnothing
$$

Significa que existem competências fornecidas pelo Método $\mathrm{ERi}^{*} \mathrm{c}$ que não fazem parte do Framework i*, mas são consideradas úteis pelos engenheiros de requisitos, cujo detalhamento deve ser modificado.

Cabe ressaltar que essa avaliação está considerando o Método ERi*c sem a etapa de diagnósticos porque a mesma não tinha sido apresentada aos participantes no treinamento do Método ERi*c.

\section{II.2 Descrição da instrumentação}

Em cada experimento estudado e para cada competência fornecida, foram oferecidas as escolhas definidas a seguir.

Resultado: $\mathrm{N}$ competências com valores (P; U; $\mathbf{A})$ onde:

$$
\begin{aligned}
& \mathbf{P}-\mathbf{P r e s e n c ̧ a ~}\{0 \rightarrow \text { não fornecida; } 1 \rightarrow \text { fornecida }\} \\
& \mathbf{U} \text { - Utilidade }\{0 \rightarrow \text { não útil; } 1 \rightarrow \text { útil }\} \\
& \mathbf{A}-\mathbf{A d e q u a c ̧ a ̃ o}\{0 \rightarrow \text { sem modificação; } 1 \rightarrow \text { com modificação }\}
\end{aligned}
$$

\section{II.3 Seleção do contexto}

O contexto é caracterizado por quatro dimensões. Nosso estudo teve o processo on-line porque os participantes responderam ao questionário da entrevista ainda durante o período do curso na universidade, no transcurso da experimentação. Os participantes 
foram todos alunos. $\mathrm{O}$ estudo é considerado modelado porque os alunos aplicaram os métodos em um problema de uma empresa de seguros fictícia. A dimensão de generalidade é considerada específica, pois a experimentação foi sobre o contexto específico de uma modelagem de sistema orientado a metas.

\section{II.4 Seleção dos participantes}

Como participantes da experimentação tivemos alunos da graduação da UERJ e alunos da graduação da PUC-Rio da área de Engenharia de Software. Os alunos foram consultados e avisados que se tratava de uma experimentação científica. Somente aqueles que concordaram em participar do experimento foram incluídos na pesquisa.

No primeiro experimento participaram alunos de duas turmas da UERJ e de uma turma da PUC-Rio. Não houve distinção de qual turma o aluno pertencia, eles foram considerados como um único grupo de participantes. No segundo e no terceiro experimentos participaram exclusivamente alunos de uma turma da UERJ, somente para esta turma foi ensinado o Método ERi*c.

\section{II.5 Definição das variáveis}

\section{II.5.1 Definição das variáveis para a experimentação 1}

As variáveis independentes se referem à entrada da experimentação, são elas:

- a lista das competências fornecidas pelo Framework i*.

As variáveis dependentes se referem à saída da experimentação, são elas:

- o grau da similaridade entre as competências fornecidas pelo Framework $i^{*}$ e as competências anteriormente assimiladas;

- o grau da utilidade das competências fornecidas pelo Framework i* e as competências anteriormente assimiladas;

- o grau de adequação das competências fornecidas pelo Framework i*.

\section{II.5.2 Definição das variáveis para a experimentação 2}

As variáveis independentes se referem à entrada da experimentação, são elas:

- a lista das competências fornecidas pelo Método ERi*c.

As variáveis dependentes se referem à saída da experimentação, são elas:

- o grau da similaridade entre as competências fornecidas pelo Método ERi*c e as atribuídas ao Framework i*;

- o grau da utilidade das competências fornecidas pelo Método ERi*c e as competências atribuídas ao Framework i*;.

- o grau de adequação das competências fornecidas pelo Método ERi*c. 


\section{ETAPAS DE EXECUÇÃO E DE ANÁLISE}

Os experimentos foram executados em seqüência, eles aconteceram em períodos distintos. Todos os treinamentos foram exatamente iguais, foram ministrados pelo mesmo professor, com o mesmo material didático e com a mesma carga horária. Cada experimentação teve um questionário específico conforme está apresentado nas seções seguintes.

\section{III.1 Operacionalização e análise da experimentação 1}

\section{III.1.1 - Questionários das Competências e do Perfil do Participante}

O questionário completo da experimentação do Framework i* encontra-se em [Oliveira, 2008a] para a "Avaliação da Aplicação do Framework i* - Caso da Seguradora Imperial". Uma descrição resumida do conteúdo das competências é apresentada no Quadro 1.

Quadro 1. Quadro resumo das competencias avaliadas na Experimentação 1

\begin{tabular}{|c|c|c|}
\hline & COMPETÊNCIA & DEsCRIÇÃo \\
\hline $\mathbf{A}$ & Identificação de atores & $\begin{array}{l}\text { Com a utilização do } i^{*} \text { você é capaz de identificar os } \\
\text { atores de uma organização. }\end{array}$ \\
\hline B & $\begin{array}{l}\text { Identificação de } \\
\text { dependências }\end{array}$ & $\begin{array}{l}\text { Com a utilização do i* você é capaz de identificar as } \\
\text { dependências dos atores de uma organização. }\end{array}$ \\
\hline $\mathbf{C}$ & $\begin{array}{l}\text { Identificação de } \\
\text { oportunidades }\end{array}$ & $\begin{array}{l}\text { Com a utilização do } i^{*} \text { você é capaz de identificar as } \\
\text { oportunidades dos atores de uma organização. }\end{array}$ \\
\hline D & $\begin{array}{l}\text { Elicitação de metas } \\
\text { concretas }\end{array}$ & $\begin{array}{l}0 \text { i* é capaz de descobrir as metas concretas dos } \\
\text { atores de uma organização. }\end{array}$ \\
\hline $\mathbf{E}$ & $\begin{array}{l}\text { Elicitação de metas } \\
\text { flexíveis }\end{array}$ & $\begin{array}{l}0 \text { i* é capaz de descobrir as metas flexíveis dos } \\
\text { atores de uma organização. }\end{array}$ \\
\hline $\mathbf{F}$ & Representação de metas & $\begin{array}{l}0 \mathrm{i}^{*} \text { é capaz de representar as metas concretas e } \\
\text { as metas flexíveis dos atores. }\end{array}$ \\
\hline $\mathbf{G}$ & $\begin{array}{l}\text { Identificação de agentes, } \\
\text { posições e papéis }\end{array}$ & $\begin{array}{l}0 i^{*} \text { é capaz de identificar os agentes, as posições } \\
\text { e os papeis dos atores. }\end{array}$ \\
\hline $\mathbf{H}$ & $\begin{array}{l}\text { Representação de agentes, } \\
\text { posições e papéis }\end{array}$ & $\begin{array}{l}0 \text { i* é capaz de representar nos modelos os } \\
\text { agentes, as posições e os papeis dos atores. }\end{array}$ \\
\hline $\mathbf{I}$ & Análise de variabilidades & $\begin{array}{l}O \mathrm{i}^{*} \text { é capaz de fazer análise de alternativas para a } \\
\text { fase de desenho (design) do software. }\end{array}$ \\
\hline J & Niveis de detalhamento & $\begin{array}{l}0 \mathrm{i}^{*} \text { permite o engenheiro de requisitos fazer } \\
\text { modelos com níveis de detalhamento. }\end{array}$ \\
\hline $\mathbf{K}$ & $\begin{array}{l}\text { Simplificação da } \\
\text { complexidade }\end{array}$ & $\begin{array}{l}0 \mathrm{i}^{*} \text { permite o engenheiro de requisitos fazer } \\
\text { modelos de complexidade simples. }\end{array}$ \\
\hline $\mathbf{L}$ & $\begin{array}{l}\text { Utilização de blocos } \\
\text { construtores }\end{array}$ & $\begin{array}{l}0 \mathrm{i}^{*} \text { permite nos modelos SD e SR construir blocos } \\
\text { construtores. }\end{array}$ \\
\hline $\mathbf{M}$ & $\begin{array}{l}\text { Guia para elaboração dos } \\
\text { modelos }\end{array}$ & $\begin{array}{l}O \mathrm{i}^{*} \text { possui um guia para orientar o engenheiro } \\
\text { de requisitos na elaboração dos modelos. }\end{array}$ \\
\hline
\end{tabular}

\section{III.1.2 Resultados do Estudo}

No Quadro 2 são listados os resultados dos questionários sem tratamento estatístico. Foram recebidas 17 respostas de participantes para a experimentação 1. 
Quadro 2. Resumo dos Resultados do Questionário da Experimentação 1

\begin{tabular}{|c|l|c|c|c|}
\hline \multicolumn{2}{|c|}{ Competência } & P & U & A \\
\hline A & Identificação de atores & $3: 14$ & $0: 17$ & $4: 13$ \\
\hline B & Identificação de dependências & $9: 8$ & $0: 17$ & $6: 11$ \\
\hline C & Identificação de oportunidades & $17: 0$ & $0: 17$ & $13: 4$ \\
\hline D & Elicitação de metas concretas & $9: 8$ & $0: 17$ & $9: 8$ \\
\hline E & Elicitação de metas flexíveis & $5: 12$ & $0: 17$ & $10: 7$ \\
\hline F & Representação das metas & $5: 12$ & $0: 17$ & $5: 12$ \\
\hline G & Identificação de agentes, papéis e posições & $6: 11$ & $0: 17$ & $9: 8$ \\
\hline H & Representação de agentes, papéis e posições. & $7: 10$ & $0: 17$ & $11: 6$ \\
\hline I & Análise de variabilidades & $15: 2$ & $0: 17$ & $12: 5$ \\
\hline J & Níveis de detalhamento & $11: 6$ & $0: 17$ & $10: 7$ \\
\hline K & Simplificação da complexidade & $10: 7$ & $0: 17$ & $7: 10$ \\
\hline L & Utilização de blocos construtores & $13: 4$ & $0: 17$ & $11: 6$ \\
\hline M & Guia para elaboração dos modelos & $13: 4$ & $0: 17$ & $11: 6$ \\
\hline
\end{tabular}

Uma análise superficial das respostas do Quadro 2, que grupou as respostas como $\mathbf{n}=$ negativa $: \mathbf{p}=$ positiva, nos permite verificar os problemas:

- Seis competências $[\mathrm{C}],[\mathrm{I}],[\mathrm{J}],[\mathrm{K}],[\mathrm{L}]$ e $[\mathrm{M}]$ tiveram claramente o reconhecimento da presença "P" contestado pelos participantes. Uma sinalização importante para essas competências foi o reconhecimento unânime da utilidade delas pelos entrevistados, apesar das mesmas não terem a presença reconhecida pela maioria.

- O atributo "A" adequação que aborda o detalhamento de como as competências são tratadas pelo Framework $i^{*}$ mostra que as respostas não possuem predominância positiva ou negativa. Duas possibilidades: ou o treinamento foi insuficiente ou o Framework $i^{*}$ não fornece detalhes para as competências de interesse.

- Ainda para as competências $[\mathrm{I}]$ e $[\mathrm{L}]$, o atributo "A" mostra que o número de participantes que reclamaram do detalhamento é maior que o número de participantes que reconheceram a presença "P" da competência. A análise das respostas também pode indicar que as perguntas foram mal formuladas ou mal explicadas, pois as respostas ficaram sem sentido. São os casos das competências: [I] Análise de variabilidades e [L] Utilização de blocos construtores.

O tempo de aprendizado do treinamento foi de 4 horas, três aulas de aproximadamente 80 minutos. Ele foi considerado insuficiente pelos participantes. A maioria dos participantes precisou de pelo menos mais 3 horas estudando o material fornecido.

\section{III.1.3 Análise e Interpretação dos Resultados da Experimentação 1}

\section{III.1.3.1 Validação dos Dados da Experimentação 1}

Não foram observadas respostas não válidas, as quais estariam erradas do ponto de vista dos valores lógicos válidos. Por exemplo, a resposta concomitante de negação da 
presença de alguma competência com a negação da utilidade da competência em conjunto com a indicação da necessidade de modificação da competência não ocorreu.

\section{III.1.3.2 Aplicação do teste estatístico}

Para cada competência foi aplicado o teste qui-quadrado para definir:

- se pode ser considerado que a competência é fornecida;

- se pode ser considerado que a competência é útil;

- $\quad$ se pode ser considerado que a competência não necessita detalhamento.

A matriz do Quadro 3 apresenta o comportamento fictício para $\mathbf{p}$ respostas positivas e $\mathbf{n}$ respostas negativas enquanto a distribuição ideal aconteceria sem a ocorrência de nenhuma resposta negativa.

Quadro 3. Comportamento fictício

\begin{tabular}{|c|c|c|c|}
\hline \multirow{2}{*}{ DISTRIBUIÇÃO } & \multicolumn{2}{|c|}{ RESPOSTA } & \multirow{2}{*}{ TOTAL } \\
\cline { 2 - 3 } & positiva & negativa & \\
\hline IDEAL & 17 & $\mathbf{0}$ & 17 \\
\hline REAL & $\mathbf{p}$ & $\mathbf{n}$ & 17 \\
\hline TOTAL & $\mathbf{1 7 + p}$ & $\mathbf{n}$ & $\mathbf{3 4}$ \\
\hline
\end{tabular}

Determinação do grau de liberdade (d. f.): Grau de liberdade representa o numero de variáveis independentes que fazem parte do estudo. Como os dois grupos, ou as duas variáveis, em estudo (o ideal e o real) são independentes, o grau de liberdade (d. f.) é 2 (dois). Para o nível de significância de 0,05 , que representa a probabilidade de aceitar a hipótese nula (H0) quando ela é falsa, obtemos da tabela de valores críticos de qui-quadrado [Box 78]. Valor crítico $=($ d.f. $=2, \alpha=0,05)=\underline{\mathbf{5 , 9 9 1}}$.

\section{III.1.3.3 Memória de Cálculo dos Valores do qui-quadrado}

Na matriz do Quadro 4, oij são as freqüências obtidas nas respostas do questionário, eij são as freqüências esperadas e oit é o total na linha i.

Quadro 4. Freqüências obtidas nas respostas

\begin{tabular}{|c|c|c|c|c|c|}
\hline \multirow{2}{*}{ DISTRIBUIÇÃO } & \multicolumn{4}{|c|}{ RESPOSTA } & \multirow{2}{*}{ TOTAL } \\
\cline { 2 - 5 } & \multicolumn{2}{|c|}{ positiva } & \multicolumn{2}{c|}{ negativa } & \\
\hline IDEAL & $\mathrm{o} 11$ & $\mathrm{e} 11$ & $\mathrm{o} 12$ & $\mathrm{e} 12$ & $\mathrm{o} 1 \mathrm{t}$ \\
\hline REAL & $\mathrm{o} 21$ & $\mathrm{e} 21$ & $\mathrm{o} 22$ & $\mathrm{e} 22$ & $\mathrm{o} 2 \mathrm{t}$ \\
\hline TOTAL & \multicolumn{2}{|c|}{ ot 1} & \multicolumn{2}{|c|}{ ot 2} & $\mathrm{~T}$ \\
\hline
\end{tabular}

Freqüências esperadas: $\mathrm{eij}=($ total da linha $\mathrm{i} \times$ total da coluna $\mathrm{j}) / \mathrm{T}$.

Cálculo aplicando o qui-quadrado para a observação (5:12)

$\mathrm{e} 11=(29 \times 17) / 34=14,5 \mathrm{e} 12=(5 \times 17) / 34=2,5$

$\mathrm{e} 21=(29 \times 12) / 34=14,5 \mathrm{e} 22=(5 \times 17) / 34=2,5$ 


$$
\begin{aligned}
& \text { qui-quadrado }=\sum(\text { oij }- \text { eij })^{2} / \text { eij }= \\
& =(17-14,5)^{2} / 14,5+(12-14,5)^{2} / 14,5+(0-2,5)^{2} / 2,5+(5-2,5)^{2} / 2,5= \\
& =0,43+0,43+2,5+2,5=\underline{\mathbf{5 , 8 6}}
\end{aligned}
$$

Como o valor calculado (5:12) 5,86 é menor que o valor crítico $5,99 \rightarrow \mathbf{H}_{0}$ não pode ser rejeitada. O que significa que a competência fornecida pelo Framework $i^{*}$ não é equivalente à competência anteriormente assimilada.

Para qualquer observação (n:p) com vantagem superior a calculada anteriormente (5:12), ou seja maior número de respostas positivas $\mathbf{p} e$ conseqüentemente menor número de respostas negativas $\mathbf{n}$, podemos, sem calcular, concluir que aquelas observações também não podem rejeitar a hipótese nula, porque todas certamente têm o valor do qui-quadrado calculado menores ainda que o valor crítico. São elas: (4:13), (3:14), (2:15), (1:16) e (0:17).

Para comprovar que o valor receberia um acréscimo e para aplicar o quiquadrado com uma fórmula simplificada, fizemos o cálculo para a observação (6:11), a qual é menos favorável que a observação (5:12): Usando a formula simplificada abaixo:

Qui-quadrado $=[(011 \times 022)-(012 \times 021)]^{2} \times \mathrm{T} /(0.1 \times 02 . \times 01 . \times 0.2)$

Qui-quadrado $=((17 \times 6)-(11 \times 0)]^{2} \times 34 /(28 \times 6 \times 17 \times 17)=\underline{\mathbf{7 , 2 8}}$

\begin{tabular}{l|c|c|c|c|c}
\hline Distribuição & $(-7:+10)$ & $(-6:+11)$ & $(-5:+12)$ & $(-4:+13)$ & $(-3:+14)$ \\
\hline Valor qui-2 & & 7,28 & 5,86 & & \\
\hline \multicolumn{5}{c}{ Valor crítico $=\underline{5,99}$}
\end{tabular}

\section{III.1.3.4 Análise dos Resultados da Experimentação}

A idéia da aplicação do teste qui-quadrado consiste na comparação da distribuição das respostas dos participantes com uma distribuição ideal. A distribuição ideal seria aquela que não recebe respostas negativas. Ou seja, a competência é recebida, é útil e não precisa de alteração no detalhamento. Essa distribuição ideal teria o comportamento representado pela expressão: $\{1,1,0\}$

Para nosso estudo estamos considerando o Framework $i^{*}$ como ideal e a comparação se faz com o real que no caso são as competências consideradas antes do treinamento do $i^{*}$.

As lacunas do Quadro 5 foram preenchidas com o valor "1" quando o resultado da aplicação do teste qui-quadrado não pode rejeitar a hipótese nula e com "0" no caso contrário.

Dentre as 13 competências investigadas observamos que 3 estão reconhecidamente presente no Framework i*, são elas A, E e F. Todas as 3 competências foram consideradas úteis e 2 dessas competências tiveram o detalhamento da competência considerado adequado pelo experimento, são elas A e F. 
Quadro 5. Resultado das Estatísticas da Experimentação 1

\begin{tabular}{|c|c|c|c|c|c|c|c|c|c|c|c|c|c|}
\hline & \multicolumn{13}{|c|}{ Competências } \\
\hline & $\mathbf{A}$ & B & C & D & $\mathbf{E}$ & $\mathbf{F}$ & G & $\mathbf{H}$ & I & J & $\mathbf{K}$ & $\mathbf{L}$ & M \\
\hline Presença & 1 & $\mathbf{0}$ & $\mathbf{0}$ & $\mathbf{0}$ & $\mathbf{1}$ & 1 & $\mathbf{0}$ & $\mathbf{0}$ & $\mathbf{0}$ & $\mathbf{0}$ & $\mathbf{0}$ & $\mathbf{0}$ & $\mathbf{0}$ \\
\hline Utilidade & 1 & 1 & 1 & 1 & 1 & 1 & 1 & 1 & 1 & 1 & 1 & $\mathbf{1}$ & 1 \\
\hline Adequação & 1 & $\mathbf{0}$ & $\mathbf{0}$ & $\mathbf{0}$ & $\mathbf{0}$ & 1 & $\mathbf{0}$ & $\mathbf{0}$ & $\mathbf{0}$ & $\mathbf{0}$ & $\mathbf{0}$ & $\mathbf{0}$ & $\mathbf{0}$ \\
\hline
\end{tabular}

Como nosso objetivo do estudo da experimentação é avaliar se o Método ERi*c promove vantagens de competências em relação ao Framework $i^{*}$ deixamos para fazer as análises quantitativas e qualitativas após a aplicação do experimento 2 .

\section{III.2 Operacionalização e análise da experimentação 2}

O Estudo das Competências do Método ERi*c

\section{III.2.1 Questionários das Competências e do Perfil do Participante}

O questionário da experimentação [Oliveira 08a] foi essencialmente o mesmo que foi aplicado na experientação 1 .

\section{III.2.2 Resultados do Estudo}

A seguir (Quadro 6) são apresentados os resultados do questionário. Foram recebidas 9 respostas dos participantes da experimentação 2 .

Quadro 6. Resumo dos Resultados do Questionário da Experimentação 2

\begin{tabular}{|c|l|c|c|c|}
\hline \multicolumn{1}{|c|}{ Competência } & P & U & A \\
\hline A & Identificação de atores & $1: 8$ & $0: 9$ & $1: 8$ \\
\hline B & Identificação de dependências & $4: 5$ & $0: 9$ & $2: 7$ \\
\hline C & Identificação de oportunidades & $9: 0$ & $2: 7$ & $7: 2$ \\
\hline D & Elicitação de metas concretas & $0: 9$ & $0: 9$ & $2: 7$ \\
\hline E & Elicitação de metas flexíveis & $3: 6$ & $0: 9$ & $3: 6$ \\
\hline F & Representação das metas & $1: 8$ & $0: 9$ & $3: 6$ \\
\hline G & Identificação de agentes, papéis e posições & $7: 2$ & $1: 8$ & $8: 1$ \\
\hline H & Representação de agentes, papéis e posições & $3: 6$ & $0: 9$ & $4: 5$ \\
\hline I & Análise de variabilidades & $6: 3$ & $5: 4$ & $9: 0$ \\
\hline J & Niveis de detalhamento & $6: 3$ & $0: 9$ & $3: 6$ \\
\hline K & Simplificação da complexidade & $3: 6$ & $3: 6$ & $3: 6$ \\
\hline L & Utilização de blocos construtores & $7: 2$ & $0: 9$ & $4: 5$ \\
\hline M & Guia para elaboração dos modelos & $6: 3$ & $0: 9$ & $7: 2$ \\
\hline
\end{tabular}

Uma análise superficial das respostas, antes da análise estatística, nos permite verificar pontos que deveriam ser melhorados no Método ERi*c e por isso demandaram alterações no detalhamento das heurísticas no método. 
Outras respostas também chamaram a nossa a atenção:

- A maioria dos participantes não reconheceu que o método favorece a [L] Utilização de blocos construtores apesar da unanimidade da resposta pela utilidade "U" dessa competência.

- A análise das respostas também permite verificar que algumas perguntas foram mal formuladas ou o treinamento não foi satisfatório, pois as respostas ficaram sem sentido para os atributos "P" e "U". São os casos das competências: [I] Análise de variabilidades teve a utilidade "U" rejeitada e [L] Utilização de blocos construtores não teve a presença "P" reconhecida.

A análise do perfil dos participantes ressaltou que o treinamento de 10 horas e 40 minutos, 8 aulas de aproximadamente 80 minutos, foi considerado insuficiente pelos participantes.

\section{III.2.3 Análise e Interpretação dos Resultados da Experimentação 2}

\section{III.2.3.1 Validação dos Dados da Experimentação 2}

Não foram observadas respostas não válidas as quais estariam erradas do ponto de vista dos valores lógicos válidos. Por exemplo, a resposta concomitante de negação da presença de alguma competência com a negação da utilidade da competência em conjunto com a indicação da necessidade de modificação da competência não ocorreu.

\section{III.2.3.2 Aplicação do teste estatístico}

Para cada competência foi aplicado o teste qui-quadrado para definir:

- se pode ser considerado que a competência é fornecida;

- se pode ser considerado que a competência é útil;

- se pode ser considerado que a competência não necessita detalhamento.

A pergunta a ser respondida pelo teste qui-quadrado é a seguinte: A partir de que valores $(\mathbf{n}=$ negativo $: \mathbf{p}=$ positivo) os resultados podem ser considerados como ideais para a distribuição com probabilidade $\alpha$ (nível de significância) de erro?

Para o nível de significância de 0.05 , que representa a probabilidade de aceitar a hipótese nula $\left(\mathrm{H}_{0}\right)$ quando ela é falsa, obtemos da tabela de valores críticos de quiquadrado $[$ Box 78]. Valor crítico $=($ d.f. $=1, \alpha=0.05)=\underline{\mathbf{3 , 8 4}}$.

$\mathbf{H}_{0}$ : Existe diferença em relação a competência fornecida pelo Método ERi*c e a competência anteriormente assimilada no Framework i*. O grau de liberdade foi considerado 1 porque o Método ERi*c é dependente do Framework i*.

Aplicando o qui-quadrado para a observação 4:5 e usando a fórmula simplificada:

\begin{tabular}{l|l|l}
\hline 9 & 0 & 9 \\
\hline $\mathbf{5}$ & $\mathbf{4}$ & 9 \\
\hline 14 & 4 & 18 \\
\hline
\end{tabular}

qui-quadrado $=[(011 \times 022)-(012 \times 021)]^{2} \times \mathrm{T} /(0.1 \times 02 . \times 01 . \times 0.2)$ 
qui-quadrado $=((9 \times 4)-(5 \times 0)]^{2} \times 18 /(14 \times 4 \times 9 \times 9)=\underline{\mathbf{5 , 1 4}}$

Aplicando o qui-quadrado para a observação 3:6

Qui-quadrado $=((9 \times 3)-(6 \times 0)]^{2} \times 18 /(15 \times 3 \times 9 \times 9)=\underline{\mathbf{3 , 6 0}}$

Aplicando o qui-quadrado para a observação 2:7

Qui-quadrado $=((9 \times 2)-(7 \times 0)]^{2} \times 18 /(16 \times 2 \times 9 \times 9)=\underline{\mathbf{2 , 2 5}}$

\begin{tabular}{c|c|c|c|c|c}
\hline Distribuição & $(-5:+4)$ & $(-4:+5)$ & $(-3:+6)$ & $(-2:+7)$ & $(-1:+8)$ \\
\hline Valor qui-2 & & 5,14 & 3,60 & 2,25 & \\
\hline
\end{tabular}

Valor crítico $=\underline{\mathbf{3 , 8 4}}$

Como o valor calculado (3:6) 3,60 é menor que o valor crítico 3,84 $\rightarrow \mathbf{H}_{\mathbf{0}}$ não pode ser rejeitada. O que significa que o Framework $i^{*}$ não é equivalente ao anteriormente assimilado para essa competência. Podemos, sem calcular concluir que as competências com vantagem superior também não podem rejeitar a hipótese nula, porque todas certamente têm o valor do qui-quadrado calculados menores ainda que o valor crítico. São elas: (1:8) e (0:9).

Para este segundo estudo estamos considerando o Método ERi*c como ideal e a comparação se faz com o real que no caso são as competências antes do treinamento do Método ERi*c.

Quadro 7. Resultado das Estatísticas da Experimentação 2

\begin{tabular}{|c|c|c|c|c|c|c|c|c|c|c|c|c|c|}
\hline & \multicolumn{13}{|c|}{ Competências } \\
\hline & $\mathbf{A}$ & B & C & D & $\mathbf{E}$ & $\mathbf{F}$ & G & H & $\mathbf{I}$ & J & $\mathbf{K}$ & $\mathbf{L}$ & $\mathbf{M}$ \\
\hline Presença & 1 & $\mathbf{0}$ & $\mathbf{0}$ & 1 & 1 & 1 & $\mathbf{0}$ & 1 & $\mathbf{0}$ & $\mathbf{0}$ & 1 & $\mathbf{0}$ & $\mathbf{0}$ \\
\hline Utilidade & 1 & 1 & 1 & 1 & $\mathbf{1}$ & 1 & $\mathbf{1}$ & 1 & $\mathbf{0}$ & $\mathbf{1}$ & 1 & 1 & 1 \\
\hline Adequação & 1 & 1 & $\mathbf{0}$ & 1 & $\mathbf{1}$ & 1 & $\mathbf{0}$ & $\mathbf{0}$ & $\mathbf{0}$ & $\mathbf{1}$ & 1 & $\mathbf{0}$ & $\mathbf{0}$ \\
\hline
\end{tabular}

As competências "C", "G", "H", "I", "L" e "M" tiveram, na época do experimento, retorno que elas precisavam um refinamento maior no detalhamento. A competência "I" teve a utilidade não reconhecida e as competências "C", "G", "L" e " $M$ " receberam retorno que são úteis e com detalhamento inadequado apesar de não estarem completamente presentes no Método ERi*c.

\section{III.2.3.3 Análise quantitativa}

Numa comparação superficial entre o FraMEwork I* e o MÉTodo ERI*C são analisadas as freqüências das competências quanto a presença, utilidade e adequação do detalhamento.

$\Rightarrow$ Métrica: Lista das competências fornecidas pelo Método ERi*c que não fazem parte do Framework i*.

$$
\mathrm{PUA}_{1 \mathbf{p}}\{1, \mathrm{X}, \mathrm{X}\}=\{\mathrm{A}, \mathrm{E}, \mathrm{F}\} \text { e } \mathrm{PUA}_{2 \mathbf{p}}\{1, \mathrm{X}, \mathrm{X}\}=\{\mathrm{A}, \mathrm{D}, \mathrm{E}, \mathrm{F}, \mathrm{H}, \mathrm{K}\}
$$


$\Rightarrow$ Métrica: Lista das competências fornecidas pelo Método ERi*c e que são consideradas inúteis pelos engenheiros de requisitos.

$$
\mathrm{PUA}_{1 \mathbf{p}}\{1,0, \mathrm{X}\}=\varnothing \text { e } \mathrm{PUA}_{2 \mathbf{p}}\{1,0, \mathrm{X}\}=\{\mathrm{I}\}
$$

$\Rightarrow$ Métrica: Lista das competências fornecidas pelo Método ERi*c que fazem parte do Framework $i^{*}$ e são consideradas úteis pelos engenheiros de requisitos, cujo detalhamento deve ser modificado.

$$
\operatorname{PUA}_{1 \mathbf{p}}\{1,1, \mathrm{X}\} \cap \mathrm{PUA}_{\mathbf{2 p}}\{1,1, \mathrm{X}\}=\{\mathrm{A}, \mathrm{E}, \mathrm{F}\}
$$

$\Rightarrow$ Métrica: Lista das competências fornecidas pelo Método ERi*c que não fazem parte do Framework i*, mas que são consideradas úteis pelos engenheiros de requisitos.

$$
\mathrm{UA}_{2 \mathbf{p}}\{1, \mathrm{X}, \mathrm{X}\}-\mathrm{PUA}_{1 \mathbf{p}}\{1, \mathrm{X}, \mathrm{X}\}=\{\mathrm{D}, \mathrm{H}, \mathrm{K}\}
$$

\section{III.4 Análise das Hipóteses}

\section{III.4.1 Análise das Hipóteses - Experimentação 1}

$\mathrm{H}_{0} \rightarrow \mathrm{O}$ resultado do questionário mostrou que 3 das 13 competências do Framework i* foram oferecidas e também foram reconhecidas como úteis. Podemos concluir que existe diferença entre a lista de competências oferecidas pelo Framework $i^{*}$ e as competências anteriormente assimiladas. (Hipótese alternativa $\mathrm{H}_{1}$ )

Dentre as competências oferecidas pelo Framework $i^{*}$ e consideradas úteis duas delas precisa de maior detalhamento. (Hipótese alternativa $\mathrm{H}_{2}$ )

\section{III.4.2 Análise das Hipóteses - Experimentação 2}

$\mathrm{H}_{0} \rightarrow \mathrm{O}$ resultado do questionário mostrou que 6 das 13 competências do Método ERi*c foram oferecidas e também foram reconhecidas como úteis. Podemos concluir que existe diferença entre a lista de competências oferecidas pelo Método ERi*c e as competências do Framework $i^{*}$ anteriormente assimiladas. (Hipótese alternativa $\mathrm{H}_{1}$ )

Dentre as competências oferecidas pelo Método ERi*c e consideradas úteis 2 delas precisavam de maior detalhamento. (Hipótese alternativa $\mathrm{H}_{2}$ )

\section{III.5 Operacionalização e análise da experimentação 3}

Faz o Estudo das Competências do "i* Diagnoses". De modo a avaliar o desempenho dos alunos na aplicação da estratégia de Diagnósticos i*, executamos um experimento com 14 participantes e fizemos a medição da eficácia do resultado.

\section{III.5.1 Questionários das Competências e do Perfil do Participante}

O questionário completo da experimentação do $i^{*}$ Diagnoses encontra-se em [Oliveira 08a] para a "Avaliação da Aplicação do i* Diagnoses - Caso Serviço de Recados na Secretaria Eletrônica". 
Descrição do experimento:

$\mathrm{O}$ experimento foi desenvolvido em duas seções. Na primeira seção foi pedido que os alunos fizessem em uma aula um Modelo SD e na aula seguinte um Modelo SR, para o caso SERVIÇO DE RECAdOS NA SECRETARIA ELETRÔNICA.

\section{Quadro 8- Quadro resumo das competencias avaliadas na Experimentação 3}

\begin{tabular}{|c|l|l|}
\hline & \multicolumn{1}{|c|}{ CompETÊNCIA } & \multicolumn{1}{c|}{ DESCRIção } \\
\hline A & $\begin{array}{l}\text { Identificação de problemas } \\
\text { com atores }\end{array}$ & $\begin{array}{l}\text { A utilização da técnica de diagnósticos é capaz de } \\
\text { identificar problemas com os atores. }\end{array}$ \\
\hline B & $\begin{array}{l}\text { Identificação de problemas } \\
\text { com dependências estratégicas }\end{array}$ & $\begin{array}{l}\text { A utilização da técnica de diagnósticos é capaz de } \\
\text { identificar os problemas com as dependências } \\
\text { estratégicas entre atores de uma organização. }\end{array}$ \\
\hline C & Identificação de oportunidades & $\begin{array}{l}\text { A utilização da técnica de diagnósticos é capaz de } \\
\text { identificar as oportunidades dos atores. }\end{array}$ \\
\hline D & Verificação de metas concretas & $\begin{array}{l}\text { A utilização da técnica de diagnósticos é capaz de } \\
\text { verificar as metas concretas dos atores. }\end{array}$ \\
\hline E & Verificação de metas flexíveis & $\begin{array}{l}\text { A utilização da técnica de diagnósticos é capaz de } \\
\text { verificar as metas flexíveis dos atores. }\end{array}$ \\
\hline F & Verificação de funcionalidades & $\begin{array}{l}\text { A utilização da técnica de diagnósticos é capaz de } \\
\text { verificar as funcionalidades representadas. }\end{array}$ \\
\hline G & $\begin{array}{l}\text { Verificação de entidades tipo } \\
\text { recurso }\end{array}$ & $\begin{array}{l}\text { A utilização da técnica de diagnósticos é capaz de } \\
\text { verificar os recursos representados. }\end{array}$ \\
\hline
\end{tabular}

Na segunda seção, a cada pessoa foi entregue dois diagramas $i^{*}$, preparados pelo avaliador, que correspondiam ao trabalho da primeira seção, porém com as correções pertinentes, e mais o framework de perguntas para diagnóstico de SDsituations e de SRconstructs. Pedimos que os participantes aplicassem as perguntas formuladas sobre os modelos SD e SR. Eles tiveram 2 horas para fazer o trabalho de diagnóstico. $\mathrm{Na}$ segunda seção a avaliação cobriu as 7 competências destacadas no formulário do Quadro 8 e, na avaliação dos trabalhos, 5 tópicos foram considerados para a avaliação da eficácia:

a) se os construtores $i *$ foram corretamente identificados;

b) se os participantes entenderam as perguntas;

c) se os participantes foram capazes de identificar as omissões;

d) se os participantes identificaram os erros em componentes e

e) se as omissões de metas flexíveis foram identificadas.

De acordo com esses tópicos o avaliador classificou os resultados como:
A - quase $100 \%$ correto
B - quase $70 \%$ correto
C - quase $50 \%$ correto e
D - menos que $40 \%$ correto 
O Quadro 9 e a Figura 1 apresentam a tabulação das notas dos trabalhos. Na segunda seção o trabalho de cada aluno foi corrigido por outro aluno que também participou do experimento usando o gabarito distribuído pelo avaliador.

Quadro 9. Mapeamento da correção do exercício SEM diagnóstico

\begin{tabular}{|c|c|c|c|c|c|c|c|c|c|c|c|c|c|c|}
\hline & \multicolumn{10}{|c|}{ Participantes } \\
\hline Competências & $\mathbf{1}$ & $\mathbf{2}$ & $\mathbf{3}$ & $\mathbf{4}$ & $\mathbf{5}$ & $\mathbf{6}$ & $\mathbf{7}$ & $\mathbf{8}$ & $\mathbf{9}$ & $\mathbf{1 0}$ & $\mathbf{1 1}$ & $\mathbf{1 2}$ & $\mathbf{1 3}$ & $\mathbf{1 4}$ \\
\hline Identificação de alternativas & $\mathbf{B}$ & $\mathbf{A}$ & $\mathbf{D}$ & $\mathbf{D}$ & $\mathbf{D}$ & $\mathbf{B}$ & $\mathbf{D}$ & $\mathbf{C}$ & $\mathbf{D}$ & $\mathbf{C}$ & $\mathbf{D}$ & $\mathbf{D}$ & $\mathbf{D}$ & D \\
\hline Identificação de problemas & $\mathbf{B}$ & $\mathbf{D}$ & $\mathbf{B}$ & $\mathbf{C}$ & $\mathbf{D}$ & $\mathbf{D}$ & $\mathbf{B}$ & $\mathbf{B}$ & $\mathbf{D}$ & $\mathbf{C}$ & $\mathbf{D}$ & $\mathbf{D}$ & $\mathbf{C}$ & D \\
\hline Verificação de metas flexíveis & $\mathbf{A}$ & $\mathbf{C}$ & $\mathbf{D}$ & $\mathbf{D}$ & $\mathbf{C}$ & $\mathbf{C}$ & $\mathbf{C}$ & $\mathbf{D}$ & $\mathbf{D}$ & $\mathbf{B}$ & $\mathbf{C}$ & $\mathbf{C}$ & $\mathbf{D}$ & D \\
\hline
\end{tabular}

A Figura 1 mostra que a estratégia teve resultado positivo em relação à eficácia. $\mathrm{O}$ resultado apontou vantagem significativa para as freqüências "A - quase $100 \%$ correto" e "B - quase $70 \%$ correto".

Os resultados da terceira experimentação, Diagnósticos i*, foram positivos:

a) a identificação dos construtores (SDsituations e SRconstructs) foi $100 \%$ alcançada por 9 dos 14 participantes;

b) o entendimento das questões foi alcançada por 8 dos 14 participantes;

c) na identificação das alternativas apenas dois participantes conseguiram $100 \% \mathrm{e}$ 11 do total de 14 participantes conseguiram resultados que fazem o modelo diagnosticado ser aprimorado. Apenas 3 participantes não chegaram a nenhum resultado proveitoso;

d) a identificação de problemas foi o item de pior desempenho: 1 aluno fez 100\%, 6 participantes quase alcançaram os $100 \%$ e 7 participantes identificaram aproximadamente metade dos problemas;

e) na identificação de metas flexíveis 7 de 14 participantes tiveram ótimo resultado e apenas 1 aluno não consegui fazer nada da elicitação de metas flexíveis.

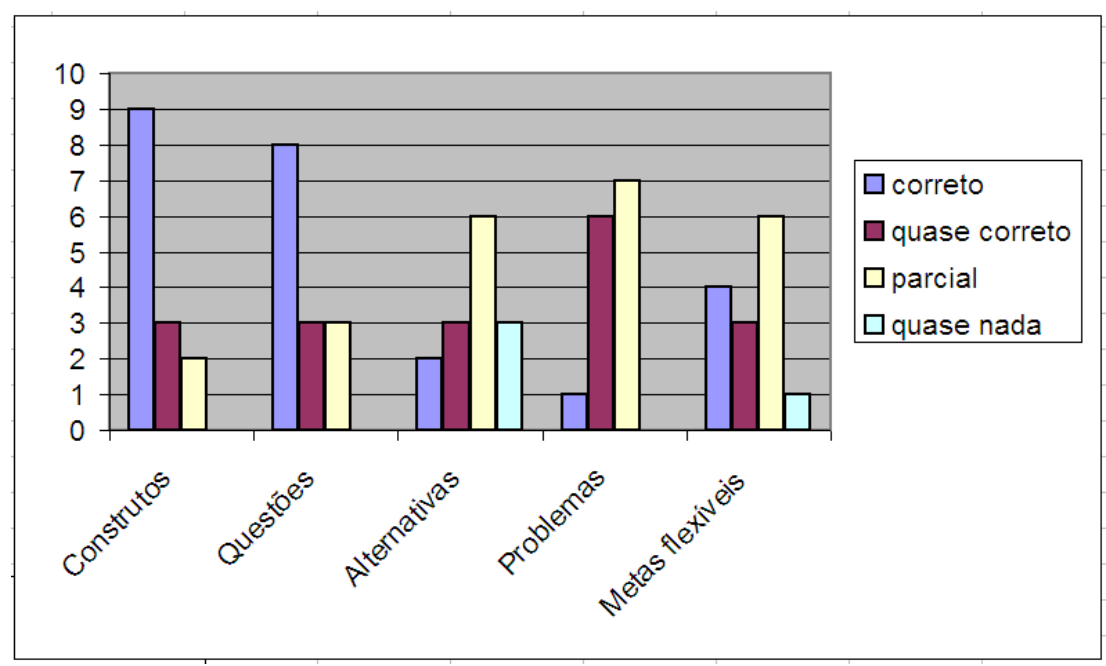

Figura 1. Gráfico de barras da correção do exercício COM diagnóstico 


\section{Conclusão}

Este artigo apresentou os fundamentos da experimentação em engenharia de software e também apresentou com detalhes três experimentos para a avaliação das competências fornecidas pelo Método ERi*c seguindo os passos do trabalho "Introdução a Engenharia Experimental" [Travassos 02].

A experimentação concluiu que o Método ERi*c contribui com a presença de 3 competências úteis $\{\mathbf{D}, \mathbf{H}$ e $\mathbf{K}\}$, além das $3\{\mathbf{A}, \mathbf{E}$ e $\mathbf{F}\}$ que o Framework i* já fornece para a engenharia de Requisitos de Sistemas Multi-Agentes. Resultado: contribuição adicional de $23 \%$.

Como resultado as experimentações indicaram também que o Método ERi*c possuía problemas de clareza e de detalhamento nas heurísticas da utilização do método mas, mesmo assim, o Método ERi*c agregou com detalhamento adequado em 5 competências $\{\mathbf{B}, \mathbf{D}, \mathbf{E}, \mathbf{J}$ e $\mathbf{K}\}$ além das $2\{\mathbf{A}$ e $\mathbf{F}\}$ que o Framework i* já fornece. Resultado: contribuição adicional de $38 \%$.

A terceira experimentação avaliou a técnica de Diagnósticos $i^{*}$ e inferiu que com o apoio das perguntas os participantes:

- na identificação das alternativas a melhora foi de $21 \%$ ( 3 OK contra 11) para $79 \%$ (11 do total de 14 participantes conseguiram resultados);

- na competência identificação de problemas a melhora dos participantes foi de $8 \%$ para $100 \%$ ( todos os alunos encontraram pelo menos metade dos problemas);

- na identificação de metas flexíveis a melhora no desempenho dos participantes foi de $16 \%$ ( 2 OK contra 12) para $92 \%$ ( $13 \mathrm{em} \mathrm{1).}$

\section{Referências}

Basili, Victor R.; Caldiera, Gianluigi; Rombach, H. Dieter, "The Goal Question Metric Paradigm", Encyclopedia of Software Engineering (Marciniak, J.J., editor), Volume 1, John Wiley \& Sons, 1994, pp. 578-583

Basili, V. "The Role of Experimentation in Software Engineering: Past, Current, and Future," Proceedings of the Eighteenth International Conference on Software Engineering (ICSE), Berlin, Germany, March 1996.

Box, G. E. P.; Hunter, W. G., and Hunter, J. S., Statistics for experimenters. An introduction to design, data analysis and model building. New York, Wiley, 1978.

Estrada, H; Martínez, A; Pastor, O; Mylopoulos, J.; An Experimental Evaluation of the i* Framework in a Model-based Software Generation Environment; E. Dubois, K. Pohl (Eds.); CAISE 06, LNCS vol. 4001, pp.513-527, Springer-Verlag, Berlin Heidelberg, ISBN: 3-540-34652-X, 978-3-540-34652-4 - 2006.

Mafra, Sômolo; Travassos, Guilherme; "Estudos Primários e Secundários apoiando a busca por Evidência em Engenharia de Software" - Relatório Técnico: RT-ES687/06 - Programa de Engenharia de Sistemas e Computação - COPPE/UFRJ - Rio de Janeiro, 2006. 
Oliveira, A. Padua A.; Engenharia de Requisitos Intencional: Um Método de Elicitação, Modelagem e Análise de Requisitos. PhD Tese, PUC-Rio, Março/2008.

Oliveira, A. Padua A.; Leite, J. C. S. P.; Cysneiros, L. M.; Lucena, C. J.; i* Diagnoses: A Quality Process for Building i* Models - Proceedings of the Forum at the CAiSE'08, Montpellier, France, June 18-20, 2008. CEUR Workshop Proceedings 344 CEUR-WS.org 2008 pp. 9-12.

Pastor, Oscar; Estrada, Hugo; Martínez, Alicia; The Strengths and Weaknesses of the i* Framework: an experimental evaluation $i^{*}$, its Applications, Variations and Extensions. Eric Yu et als. (eds.) 2006 - MIT Press.

Travassos, Guilherme; Gurov, Dmytro; Amaral, Edgard; "Introdução a Engenharia de Software Experimental" - Relatório Técnico: RT-ES-590/02 - Programa de Engenharia de Sistemas e Computação - COPPE/UFRJ - Rio de Janeiro, 2002.

$\mathrm{Yu}$, E. Modelling Strategic Relationships for Process Reengineering. PhD Thesis, Graduate Department of Computer Science, University of Toronto, Canada, 1995. 